\title{
Current Views on Surgical Treatment of Pancreatic Cancer
}

\author{
Hugo Obertopa Sergio Pedrazzoli ${ }^{\text {b }}$ \\ aDepartment of Surgery, Academic Medical Center, University of Amsterdam, The Netherlands, and \\ bDipartimento di Scienze Mediche e Chirurgiche, Semeiotica Chirurgica, Università di Padova, Italia
}

Surgery is still the cornerstone of treatment of pancreatic cancer. Radical resection offers the only opportunity to cure patients with pancreatic ductal adenocarcinoma. However, resection rates are not higher than $15 \%$ because most patients have advanced and therefore incurable disease at the time of diagnosis.

Obstructive jaundice is the most frequent presenting symptom because the majority of cancers are located in the pancreatic head. Pain, weight loss and gastric outlet obstruction are later symptoms. Relief of biliary obstruction and the concurrent anorexia and pruritus is mostly performed as a first step and is especially indicated in frail deeply jaundiced patients. Endoscopic insertion of an endoprosthesis after diagnostic ERCP can be performed as a preoperative measure in order to improve the patient's condition and to bridge the time for operation, although the beneficial effect shown in experimental animals could not be confirmed in controlled clinical trials. Furthermore the role of ERCP as a diagnostic tool is limited. When incurable disease is diagnosed after further staging procedures, an endoprosthesis may provide definite palliation, although irresectable patients with a relatively good prognosis may benefit from surgical bypass. In further dianosis and staging spiral CT scanning and US with Doppler play an important role.

In those patients that are considered resectable, a diagnostic laparoscopy with laparoscopic US is sensitive for the detection of peritoneal deposits and local tumor ingrowth; although the yield (i.e. prevention of unnecessary surgical explorations) is not more than $15 \%$ and not

\begin{tabular}{ll}
\hline KARGER & ( 1999 S. Karger AG, Basel \\
0253-4886/99/0164-0263\$17.50/0 \\
$\begin{array}{l}\text { Fax +4161306 12 34 } \\
\text { E-Mail karger@karger.ch } \\
\text { www.karger.com }\end{array}$ & $\begin{array}{l}\text { Accessible online at: } \\
\text { http://BioMedNet.com/karger }\end{array}$
\end{tabular}

cost-effective in pancreatic head tumors, it has a much higher yield in those few adenocarcinomas of the pancreatic body and tail that are considered resectable. Even after extensive staging, surgical exploration will show irresectable tumor in more than half of the patients. In case of irresectability, hepaticojejunostomy is the bypass of choice and frequently a (prophylactic) gastrojejunostomy is added, although evidence to support this policy is lacking. Peroperative chemical blockage of the coeliac plexus should be added, as this has been shown to be beneficial in one prospective randomized trial.

For those patients with resectable pancreatic head tumors, pancreatoduodenectomy (named after Kausch and Whipple) is indicated and distal pancreatectomy for resectable body and tail tumors.

Pancreatic cancer is judged to be resectable when no distant metastases or gross local tumor ingrowth is seen. In the case of limited tumor ingrowth in the portal or superior mesenteric vein, partial resection of these structures and subsequent reconstruction can be performed, leading to a somewhat higher operative risk and decreased survival in most series.

The pylorus preserving pancreatoduodenectomy is preferred for the treatment of pancreatic head tumor and will provide better long-term gastrointestinal function without jeopardizing the chance of cure. No uniformity exists on the technique of the operation and extent of lymph node dissection during pancreatectomy and there is no standardization of the nomenclature. 
In this issue of Digestive Surgery the results of a consensus meeting of surgeons and pathologists are reported. Consensus was reached on the nomenclature of three types of the Kausch-Whipple operations: standard, radical and extended radical pancreatoduodenectomy. The difference is the extent of lymph node dissection. The Japanese classification system is used to refer to the various groups of lymph nodes. The use of this terminology and adherence to one of the three described techniques will make a comparison of the results from different surgeons possible.

The superiority of radical (or extended radical) procedures, with a more extended lymph node dissection, over standard pancreatoduodenectomy has not yet been clearly proved. In one prospective study comparing these two techniques, no overall survival benefit was shown. A survival benefit was found, using a posteriori analysis, in a subgroup with positive lymph nodes. Pancreatoduodenectomy can be carried out with a very low mortality in high volume hospitals, performing 20 or more pancreatoduodenectomies per year.
The quality of life is reported to be good 3 months after pancreatoduodenectomy and even palliative resections are performed (i.e. resections with positive resection margins, usually after microscopic examination). Long-term survival after pancreatoduodenectomy for pancreatic head carcinoma is not higher than $20 \%$ in most series and after pancreatectomy for body and tail cancers reports of long-term survival are only anecdotal. Adjuvant radiochemotherapy has to date not been shown to prolong survival after resection and new chemotherapeutic drugs and regimens are currently under study.

Cost-effective preoperative diagnosis and staging leading to selection of patients that could benefit from resection and the best palliative support in all others, all performed in a well-standardized manner with low mortality and morbidity, is the best we can do. Even then surgery plays a minor role in the cure and novel therapies including immunotherapy and gene therapy should be explored for this dismal disease. 